\title{
La creación de la policía barrial en Córdoba, Argentina. ¿Una disputa en torno al ideal policial?*
}

DOI: https://doi.org/10.18046/recs.i31.3711

The Creation of the Neighborhood Police in Córdoba, Argentina. A Dispute over the Police Ideal?

\section{Déborah Goldin ${ }^{* *}$}

Universidad Nacional de Villa María (Villa María, Argentina)

\footnotetext{
* Esta investigación se materializó en la escritura de mi trabajo final de grado en la Licenciatura en Sociología (UNVM), dirigida por la magíster Mariana Carbajo y el doctor Juan Manuel Reynares. Asimismo, fue financiada parcialmente por dos becas de Estímulo a las Vocaciones Científicas (EVC) otorgadas por el Consejo Interuniversitario Nacional de Argentina (CIN). En adición, esta indagación forma parte de un proyecto de investigación titulado "Políticas de seguridad y policía en la provincia de Córdoba: continuidades y rupturas a partir del Plan Integral de Seguridad Ciudadana y Prevención del delito (2015-2017)", dirigido por el doctor Marcelo Saín, codirigido por Mariana Carbajo y financiado por la Universidad Nacional de Villa María. Artículo de investigación recibido el 15.09.2O19 y aceptado el 17.03.2O2O.

** Licenciada en Sociología de la Universidad Nacional de Villa María (Argentina). Becaria doctoral de CONICET en el Instituto Académico Pedagógico de Ciencias Sociales de la Universidad Nacional de Villa María (Argentina). Correo electrónico: debigoldin@gmail.com ORCID: https://orcid.org/oooo-ooo2-0338-9109
} 


\section{Cómo citar/How to cite}

Goldin, Déborah (2020). La creación de la policía barrial en Córdoba, Argentina. ¿Una disputa en torno al ideal policial? Revista CS, 31, 243-268. https://doi.org/10.18046/recs.i31.3711 


\section{Resumen}

Este artículo aborda las implicancias de la incorporación del Programa de Policía Barrial en los procesos de identificación de los(as) suboficiales de la Policía de Córdoba, Argentina (2016-2017). Asimismo, se pretende analizar los modos en los que la incorporación de esta nueva dependencia habilita corrimientos con respecto al ideal policial tradicional. Se considera relevante el análisis de las recepciones y los efectos de las iniciativas gubernamentales en los miembros de la institución policial, en tanto agencia privilegiada en la puesta en acto de estas políticas de prevención del delito. La metodología de la investigación es cualitativa, centrada en análisis de fuentes secundarias y entrevistas exploratorias semiestructuradas a policías operativos. Se desarrollan las principales características de este programa y los cambios en las condiciones laborales de quienes se unieron a esta dependencia. Así, se problematiza cuáles son los modos de intervención policial legítimos y las aptitudes deseables para ser policía.

PALABRAS CLAVE:

policía barrial, ideal policial, policiamiento de proximidad, condiciones laborales

This article analyses the implications of the Neighborhood Police Program incorporation in the identification processes of the Córdoba Police Department's lower ranks in Argentina (2016-2017). Thus, the paper analyzes the ways in which the incorporation of this new unit enables landslides regarding the traditional police ideal. Considering the Police force as a key agency in the implementation of new crime prevention policies, this study considers the importance of analyzing the effects of government initiatives on the members of the institution as a starting point. It is a qualitative research based on the analysis of secondary sources and exploratory, semistructured interviews to police officers in operative areas. Here, the main characteristics of this program and the changes in the labor conditions for those that began to be part of this unit are described. Furthermore, the ways of police intervention legitimated and the desirable aptitudes for a policeman are also problematized.

\section{KEYWORDS:}

Neighborhood Police, Police Ideal, Proximity Policing, Labor Conditions 



\section{Introducción}

El presente artículo se propone analizar la implementación del Programa de Policía Barrial (2016-2017) en la Provincia de Córdoba (Argentina), haciendo hincapié en sus implicancias en los procesos de identificación del personal subalterno de la Policía de Córdoba. En particular, se problematizarán algunos corrimientos con respecto al ideal policial tradicional, que son habilitados por los cambios en las condiciones laborales y las formas de trabajo de esta dependencia. De este modo, se realizará un estudio de caso del proceso de implementación de la policía barrial, con especial atención a los efectos que tuvo para el personal policial su incorporación a la misma.

La discusión que aquí se desarrollará forma parte de una indagación sobre los procesos de identificación policiales en Córdoba, y es por ello que se enfatiza el análisis de las implicancias de la creación de esta nueva dependencia en las identidades policiales, problematizando las tensiones generadas en torno al ideal policial. En esta línea, la perspectiva de los actores policiales tendrá un lugar de predominancia relativa al tratarse de un estudio centrado en los procesos de identificación policiales y no en las particularidades de la implementación de una política pública en materia de seguridad.

$\mathrm{Al}$ interior de la frondosa producción académica a nivel regional sobre (in)seguridad, violencias y delito, esta investigación se ubica de manera más específica en lo que podría llamarse una sociología de la policía. Particularmente, se propone una problematización de las identidades policiales inserta en un escenario de interlocuciones vinculado a la cuestión securitaria.

De este modo, esta indagación cobra relevancia al insertarse en un contexto regional en el que tanto la problemática de la (in)seguridad como las respuestas estatales a la misma asumen una notable centralidad en la agenda pública. En Latinoamérica, la (in)seguridad se ha instalado como una de las principales demandas ciudadanas (Dammert, 2007), asumiendo centralidad entre los tópicos de debate político. En el caso de Argentina, la (in)seguridad (hegemonizada por su vinculación con el delito común) comienza a ocupar una mayor centralidad en la agenda pública a partir de fines de los años noventa (Sozzo, 2014) y principio de los 2000 (Kessler, 2011). A partir de aquí-aunque no sin vaivenes-, ha logrado mantenerse en agenda, ocupando un lugar central entre las preocupaciones públicas, de la mano de actores políticos y mediáticos que conforman un circuito de legitimación del problema (Lorenc-Valcarce, 2009). Este proceso se da en paralelo con una creciente electoralización del tratamiento de la seguridad (Sozzo, 2008), lo que deriva en pronunciaciones recurrentes por parte de las autoridades de gobierno y decisores políticos en la materia. 
En este contexto, son precisamente las policías quienes, entre todas las agencias del sistema penal, se instalan como las instituciones privilegiadas para garantizar seguridad y mantener el orden público ${ }^{1}$. El foco de este estudio son los procesos de identificación policiales, por considerar que los procesos subjetivos de quienes aplican las políticas de seguridad han sido desatendidos en una serie de intelecciones sobre la policía e incluso en proyectos de reformas de la institución. De este modo, otorgar centralidad a las identidades de aquellas "burocracias hacedoras de políticas" (Seri, 2011) se torna indispensable a la hora de diseñar nuevas políticas de seguridad ciudadana o prevención del delito. Más aún, teniendo presente que los agentes pueden presentar diversas resistencias a las distintas iniciativas políticas.

A la vez, este crecimiento sostenido de la preocupación en torno a la (in)seguridad se desenlaza en contigüidad con un aumento de la punitividad en los diversos países de América Latina. Sin desconsiderar las especificidades nacionales y regionales, Sozzo (2016) presenta el aumento de las tasas de encarcelamiento en la región como indicador para dar cuenta del llamado giro punitivo. No obstante esta tendencia, aquí se considera relevante analizar la existencia de diversos matices al interior de este trazo general; es decir, reivindicar el estudio de casos que, en determinados contextos locales o temporales, den cuenta de la existencia de corrimientos con respecto a esta tendencia.

Si se asume la existencia de un espectro de posibilidades entre las políticas de tolerancia cero o manoduristas y aquellas ubicadas bajo el paraguas de la seguridad ciudadana, la implementación del Programa de Policía Barrial en la Provincia de Córdoba puede ser analizada al interior de esta tensión. Este programa tiene fuertes similitudes con las propuestas de policías comunitarias y es aplicado en la segunda ciudad con más población de Argentina, en un escenario nacional de profundización represiva ${ }^{2}$. Como se desarrollará, la creación de la policía barrial trajo consigo una mayor policialización -en tanto mayor presencia policial en los territorios como garante de determinadas relaciones sociales- pero, a la vez, postula algunos desplazamientos sobre el tipo de policiamiento propuesto. De este modo, cobra relevancia analizar las especificidades y modulaciones que este asume.

Como adelanto de sentido, es posible sostener que la policía barrial supone tensiones en torno al ideal policial y una disputa sobre cuáles son las formas legítimas

1. Actualmente, existen en Argentina cuatro policías federales (Policía Federal, Gendarmería Nacional, Prefectura Naval y Policía de Seguridad Aeroportuaria), veintitrés policías provinciales (entre ellas la de Córdoba) y una municipal (Policía Metropolitana de la Ciudad de Buenos Aires).

2. A partir de 2016, se percibe un desdibujamiento entre seguridad interior y defensa, junto con una narrativa gubernamental que incentiva el hostigamiento, el abuso de la fuerza y la ejecución sumaria por parte de las fuerzas de seguridad. 
del accionar policial que van a contrapelo de una tendencia nacional vinculada a la profundización represiva. Así, no solo es importante analizar este caso por su posición divergente en relación a la tendencia nacional, sino también por su potencialidad para dar luz a las tensiones al interior de las propias instituciones policiales, permitiendo visualizar algunas de las posibilidades y dificultades presentes en los procesos de cambio o reforma policial.

En concreto, aquí se analizará la implementación del Programa de Policía Barrial y las implicancias del mismo para los procesos de identificación del personal subalterno de la Policía de Córdoba, dando cuenta de qué maneras es puesto en tensión el ideal policial tradicional. Esto forma parte de una investigación realizada para mi trabajo final de grado de la Licenciatura en Sociología, cuyo objetivo fue analizar el modo en el que se configuraron los procesos de identificación del personal subalterno de la Policía de Córdoba, en un contexto de inestabilidad institucional y volatilidad en la orientación de las políticas de seguridad (2013-2017).

La estructura del artículo consta de cuatro partes. En primer lugar, se presenta brevemente la propuesta teórica-metodológica de la investigación, recuperando algunos aportes de los estudios del área. Seguidamente, se describe el marco de emergencia del Programa de Policía Barrial en la provincia y sus principales características, a partir de un análisis normativo. En un tercer momento, se abordan los principales cambios que trajo la incorporación a esta dependencia para los(as) suboficiales, haciendo foco en sus condiciones laborales. Por último, se problematizan los conflictos internos y la construcción de alteridades que se gesta con la incorporación de la policía barrial, teniendo presentes las disputas en torno al ideal policial aquí implicadas.

\section{Consideraciones teórico-metodológicas}

Teniendo en cuenta que el análisis que aquí se presenta forma parte de una investigación más amplia, resulta pertinente explicitar, resumidamente, algunos de los lineamientos generales de la misma. El objetivo general de la indagación fue analizar el modo en el que se configuraron los procesos de identificación del personal subalterno de la Policía de Córdoba en el período 2013-2017.

El recorte temporal corresponde a tres hitos considerados relevantes: el acuartelamiento policial de 20133; el cambio de código contravencional, de Código de Faltas

3. En diciembre de 2013, la Policía de Córdoba se acuarteló en un paro policial, que implicó el abandono transitorio del Estado policial y de sus funciones, en especial las tareas de patrullaje. En ese marco se dieron saqueos en las principales ciudades de la provincia y diversas escenas de violencia entre las que 
(CDF) a Código de Convivencia Ciudadana (CCC), en 20164, y la implementación de la policía barrial en 2016-2017. Así, este recorte temporal se construye a partir de una serie de hechos traumáticos que permiten tensionar ciertos principios regentes de las políticas de seguridad y analizar los efectos de dislocación en los procesos de constitución identitaria de los(as) policías. En este sentido, los tres hitos ponen en discusión, de diversas maneras, qué es ser policía. En el caso que aquí se abordará, la incorporación de la policía barrial habilita una disputa en torno al ideal policial encarnado en la discusión sobre cuáles son los modos legítimos de intervención policial y las aptitudes deseables en un buen policía.

La metodología de la investigación es cualitativa, centrada en análisis de fuentes secundarias (producciones científicas sobre el tema, normativas y documentos oficiales) y entrevistas exploratorias semiestructuradas a policías operativos 5 . A las entrevistas y el análisis documental se adicionan una serie de contactos informales con policías y funcionarios gubernamentales. Estos se dieron durante un período de trabajo posibilitado por un convenio entre la Secretaría de Seguridad de la Provincia de Córdoba y el Observatorio de Políticas Públicas de la Universidad Nacional de Villa María (UNVM). A partir de esta experiencia, se asistió a diversas capacitaciones e instancias de formación policial, la mayoría de ellas dirigidas a personal de la policía barrial. Asimismo, en este artículo se incorporan, como insumos, diversos informes ${ }^{6}$ realizados por el equipo de trabajo de dicho Observatorio de Políticas Públicas (OPP). De este modo, a partir de las entrevistas se reconstruyó una red de significantes nodales del discurso policial.

En esta misma sintonía, resultaba especialmente interesante cómolos(as) policías experimentaban la implementación del nuevo programa; partiendo de la conjetura de que existen desacoples y distancias entre el diseño de las políticas de seguridad y los

se destacaron los linchamientos a motociclistas. Se llegó a un acuerdo salarial al día siguiente, pero los sucesos terminaron con un saldo de una víctima fatal, más de cien heridos de diversa gravedad y alrededor de mil comercios dañados.

4. La relevancia de este suceso es que el Código de Faltas era, desde la perspectiva policial, una de las principales herramientas para hacer su trabajo. Por su parte, las organizaciones de derechos humanos denunciaron el crecimiento sistemático de las detenciones arbitrarias bajo el amparo de dicho código. El Código de Convivencia Ciudadana trajo consigo algunos cambios de importancia entre los que se destacan nuevas garantías procesales, el cambio de algunas de las figuras ambiguas y la reducción de la detención preventiva a un máximo de 8 horas (Castro; Castro; Goldin, 2016).

5. Se utilizan pseudónimos y se han quitado o cambiado algunos datos personales o del lugar de trabajo que facilitarían su identificación.

6. Particularmente, se tomarán aportes de un informe sobre capacitación de líderes y equipos de gestión de la policía barrial y otro sobre los resultados de una encuesta aplicada a líderes y supervisores de la policía barrial. 
modos en los que estas son puestas en acto por los(as) policías-y otros agentes-, como también en los efectos de estas políticas en los procesos de identificación policiales. A este respecto, este artículo se adhiere a la línea propuesta por Monjardet (2010), al sostener que siempre existe una distancia-mayor o menor-entre la intención de quienes instituyen una policía y lo que esta pone en práctica.

Por su parte, se piensan las identidades como "un conjunto de prácticas sedimentadas, configuradoras de sentido" (Aboy-Carlés, 2001:54), a partir de tres dimensiones: la alteridad, la representación y la perspectiva de la tradición. A la hora de pensar en identidades policiales, resulta interesante la noción de duplicidad identitaria (Frederic, 2009) para dar cuenta de que la integridad de estos sujetos no está definida únicamente por su pertenencia a la policía, en contraste con la idea más totalizante de self policial (Sirimarco, 2009). En este marco, se pretende dar cuenta de aquellas alteridades relevantes para el ser policial, y cómo estas varían y se reconfiguran asumiendo y perdiendo importancia relativa a partir de los tres hitos seleccionados que, se supone, son relevantes para los procesos de identificación policiales. De esta manera, se busca dar lugar a un estudio dinámico que incorpore la historicidad y contingencia en el análisis de los procesos identitarios de los(as) policías, dando cuenta del carácter nunca pleno de la estructura y las identidades que en su juego se constituyen. Aquí, en el orden de la contingencia, se ubica la posibilidad de una reactivación que conmueva las identidades (Aboy-Carlés, 2001). A este respecto, en los tres hitos seleccionados y, en particular, en la implementación de la policía barrial, se abre al menos la posibilidad de que la identidad policial sea conmovida.

Por otro lado, el presente artículo propone una utilización de la noción de ideal policial(Garriga-Zucal, 2016) para poder analizar de qué modos la policía barrial posibilita o promueve corrimientos con respecto a alguna de sus características7. Por ideal policial se hace referencia a aquella idea hegemónica al interior de la institución sobre lo que debería ser un buen policía. En este sentido, se encuentra estrechamente vinculado a los mandatos institucionales y, en consecuencia, el Estado policial $^{8}$ asume aquí un lugar central. Así, se trata de un ideal operativo, masculino y

7. Aquí se trabajó con la noción de ideal policial para simplificar el argumento y no adentrarse en disquisiciones que exceden el presente artículo. Sin embargo, en la investigación esta problematización parte de un análisis de las tensiones y disputas existentes entre lo que se denominan lógicas identitarias, las cuales son construidas como categorías intermedias a partir de la red de significantes nodales del discurso policial (Foa-Torres, 2016). En concreto, se construyeron tres lógicas: la omnipolicialista, la de dignificación y la de derechos (Goldin, 2019). La noción de ideal policial aquí utilizada tiene fuertes similitudes con la categoría de lógica omnipolicialista.

8. El Estado policial, de acuerdo a la Ley 9728 es la situación jurídica que resulta de un conjunto de deberes y derechos establecidos para el personal de la Policía de Córdoba. Entre estos, se destacan el deber de actuar en cualquier momento (se esté o no en horario laboral) en defensa de la vida y la propiedad, 
asociado a las nociones de fuerza y valentía (Sirimarco, 2009). En la misma sintonía, se destacan valores institucionales como la vocación y la entrega (Galvani, 2016), junto con una sobrevalorización de la experiencia laboral de calle, en contraposición con otros saberes más teóricos o de escritorio (Garriga-Zucal; Melotto, 2013). En adición, al menos durante el período anterior a 2015, en Córdoba este ideal policial se encontraba vinculado a lógicas belicistas y militaristas que planteaban el deber policial en términos de lucha o guerra contra el delito (Carbajo, 2016; Saín, 2008). De este modo, la noción que aquí se utiliza presenta similitudes también con aquellos elementos definidos como propios de la cultura policial (Reiner, 1992). Así, se considera que el ideal policial resulta significativo-aunque de modos diversos-para todos los miembros de la institución. Esto no quiere decir que todos los efectivos se sientan identificados o se apropien de los rasgos de este ideal, pero sí que todos(as) pueden reconocer su existencia y, a partir de allí, negociar la construcción de sus distancias y corrimientos con respecto al mismo.

\section{El Programa de Policía Barrial y el policiamiento de proximidad}

El siguiente apartado analiza la policía barrial entendiéndola como parte de una serie de tres hitos considerados relevantes para los procesos de identificación policiales. Así, esta iniciativa se aborda enmarcada en un momento de fuertes convulsiones en las políticas de seguridad provinciales (2013-2017). Previo a este período, las políticas de seguridad en Córdoba se caracterizaron por un proceso de policialización de la seguridad (Carbajo, 2016), donde la policía se consolidó como actor central en la definición de la orientación e implementación de las políticas de seguridad, fortaleciendo el abordaje punitivo de la conflictividad social. Esto implicaba el reconocimiento del saber policial como el saber experto legítimo para gestionar y decidir en torno a la cuestión securitaria. El punto álgido de esta tendencia se hizo presente a partir de 2011, con la designación de policías en los cargos políticos de la cartera del gobierno provincial encargada de la seguridad. En este marco, se destaca la pervivencia de una concepción belicista y policialista de la seguridad, junto

la obligación de guardar en la vida privada el decoro correspondiente, entre otros. Se considera que esta actividad permea toda la vida del sujeto, ya que los deberes enumerados en el Art. 15 los afectan pese a estar de franco, retirados o de baja. En este sentido, es que se sostiene que ser policía prima por sobre la idea de trabajar de policía, en tanto algunas características vinculadas a la labor se prolongan más allá de lo que sería el horario laboral. Es decir, es un estado permanente del policía, de lo que se desprende la frase "se es policía las 24 horas". 
con una remilitarización burocrática de la fuerza (Hathazy, 2013). En resumen, se puede sostener que en Córdoba se vieron agudizadas algunas tendencias represivas existentes a nivel nacional, lo que trajo aparejada una fuerte movilización social como respuesta a las políticas de seguridad y prácticas policiales discriminatorias.

Ya con el primero de los hitos, en 2013, el narcoescándalo 9 y el acuartelamiento policial significaron una crisis en la política de seguridad del gobierno de la provincia y, en consecuencia, se constituyó como un punto de inflexión. Luego del mismo, se dio una profundización represiva hacia dentro y fuera de la institución. Hacia dentro, porque luego del acuartelamiento se llevó a cabo un ajuste y disciplinamiento de la institución; y hacia fuera, porque en los meses que siguieron se aumentó la presencia policial, en general, como también los operativos focalizados y espectacularizados, en particular. Por su parte, el cambio de CDF a CCC, correspondiente al segundo hito, fue gestado durante la gobernación de José Manuel de la Sota (2011-2015) y emergió en los primeros meses de la gobernación de Juan Schiaretti (2015-2019), dejando ver sus efectos en el primer año del nuevo gobierno.

A este respecto, algunos investigadores sostienen que durante la gobernación de Schiaretti entre 2015-2019, se pueden distinguir algunos cambios en la orientación de las políticas de seguridad provinciales, con la aparición de nuevos semantemas en la gestión (Crisafulli; Castro, 2017). En este marco, destacan la puesta en práctica del nuevo Código de Convivencia Ciudadana (Ley 10326); el Plan Integral de Seguridad Ciudadana y Prevención del Delito (Ley 10437, Título II), con la implementación de la policía barrial (Resolución 674/16) como principal medida, y, por último, la reforma de la Ley de Seguridad Pública y Ciudadana (Ley 10437). Entre estas medidas, aquí se abordará la implementación de la policía barrial, por considerar que interpela fuertemente los procesos de identificación policiales, en tanto pone en discusión los modos de policiamiento legítimos, así como el ideal policial.

Se considera que esta serie de medidas, entre las que se destacan los dos últimos hitos (cambio de código contravencional y creación de la policía barrial) pueden ser pensados como desplazamientos que fueron posibles en el marco inaugurado luego de la crisis policial de 2013 descrita anteriormente.

Así, el Programa de Policía Barrial para la gestión de la Seguridad Ciudadana por Cuadrantes se inserta como una de las iniciativas enmarcadas en el "Plan Integral de Seguridad Ciudadana y Prevención del Delito: Córdoba se encuentra”. Dentro del

9. En los meses previos al acuartelamiento policial se hicieron públicas una serie de irregularidades que vinculaban, entre otros, a miembros de la Dirección de Lucha Contra el Narcotráfico de la Policía de Córdoba con el mercado ilegal de drogas. Esto derivó en una fuerte deslegitimación pública de la institución y en las renuncias de las máximas autoridades del Ministerio de Seguridad de Córdoba y de la institución policial. 
Plan se estipula la creación de Consejos Barriales de Prevención y Convivencia, con sus respectivos promotores barriales, al igual que un Observatorio de Estudios para la Convivencia y Seguridad Ciudadana. Asimismo, se establece que los miembros de la policía barrial trabajarán en conjunto con los consejos barriales y sus promotores. Según Crisafulli y Castro (2017:13), esto implicó "un reposicionamiento de actores y estrategias hacia adentro de la gestión de las políticas de seguridad de la provincia", ya que se incorpora cierto saber experto mediante convenios con universidades y se da lugar a estrategias de prevención que vinculan a la seguridad con la problemática social (habitacional, educativa, de salud, etc.) (Crisafulli; Castro, 2017).

La policía barrial se estructura siguiendo el modelo de policías comunitarias, en el marco de una concepción de seguridad inscrita en la idea de seguridad humana. De acuerdo a lo estipulado en los anexos de la Resolución 674, esta división se focaliza en el trabajo preventivo, basado en la detección de factores de riesgo, y en el conocimiento e intervención sobre los conflictos de convivencia presentes en el barrio. A nivel organizativo, se inserta en las comisarías. Cada una de estas divisiones cuenta con un líder ${ }^{10}$ del cuadrante y un supervisor del mismo. A su vez, estas dos figuras se encuentran por debajo de la figura del comisario a cargo de la jurisdicción, quien es el jefe del cuadrante ${ }^{11}$.

A diferencia de lo acontecido con otras policías comunitarias que fueron diseñadas inicialmente como instituciones separadas parcial o totalmente de la policía provincial $^{12}$, la Policía Barrial de Córdoba es una división al interior de la Policía de la Provincia de Córdoba; es decir, se instala al interior del esquema organizacional de la institución. Por su parte, la Policía de Córdoba cuenta con alrededor de 20 ooo miembros y, al igual que la mayoría de las policías del país, está compuesta por una pirámide dual que separa a oficiales y suboficiales. Se trata de una institución verticalizada, de jerarquías rígidas y con un régimen disciplinario donde es central el Estado policial (Carbajo; Rodríguez; Goldin; Buzetti, 2017)). Todos sus miembros se encuentran supeditados a

10. Líderes y supervisores son las nuevas nominaciones que adquieren los(as) jefes en la policía barrial.

11. A partir de la sistematización realizada de la implementación del Programa de Policía Barrial, es posible sostener que existieron fuertes disputas respecto a quién tenía efectivamente la autoridad máxima en los diversos cuadrantes.

12. Como ejemplo, está el caso de Santa Fe, donde la policía comunitaria, creada en 2012, respondía a la Secretaría de Seguridad Pública, pero dependía de la Dirección de Proximidad Policial con autonomía del resto de la policía de la provincia y bajo una dirección civil. Asimismo, los(as) policías comunitarios tenían como base las Casas del Policía Comunitario y no las comisarías. En resumen, existía una separación con la policía tradicional inexistente en el caso de Córdoba. 
lo establecido por la Ley 9728 de personal policial y, a partir de 2014, también se ven afectados por la Ley $10187^{13}$, conocida como la Ley antimotines.

Los miembros iniciales de la policía barrial fueron seleccionados entre el personal de la Policía de Córdoba mediante un proceso que, según lo establecido por el Anexo I de la Resolución 674, contó con una valoración grupal y otra individual, teniendo en cuenta el perfil profesional deseado. En un primer momento, se seleccionaron quienes serían los 11 líderes de los primeros cuadrantes a poner en marcha, y luego se siguió incorporando otro personal de diferentes jerarquías ${ }^{14}$. Asimismo, a partir de finales de 2016 se fueron incorporando numerosos grupos de agentes recientemente egresados de la Escuela de Suboficiales Manuel Belgrano.

La capacitación que estos miembros iniciales tuvieron durante el primer año de implementación del programa contaba con 16017 módulos específicos ${ }^{15} \mathrm{y}$ se desarrollaba durante un período de tres meses. Sin embargo, con el pasar del tiempo esta capacitación se fue flexibilizando, siendo dictada de diferentes maneras de acuerdo al momento. Por su parte, aquellos miembros de la policía barrial recién egresados de las escuelas de policía recibían la misma formación inicial que todos los aspirantes a agentes y cadetes ${ }^{16}$. No obstante, a partir de 2017, en la formación inicial de todos los aspirantes a agentes se incorporó una asignatura llamada Introducción a la Policía Barrial, en la que se trabajaban los principios filosóficos del policiamiento de proximidad. Asimismo, en 2019 se creó, en la Escuela de Oficiales, una especialización en proximidad. De este modo, las capacitaciones específicas de la policía barrial se encuentran profundamente articuladas (cuando no son exactamente las mismas) con las capacitaciones que reciben todos(as) los(as) policías. Por último, se incorporaron también algunas capacitaciones especiales sobre policía barrial para la mayoría del personal jerárquico de la institución con el objetivo de facilitar

13. Esta ley, denominada Régimen de Prevención para Alteraciones en la Subordinación Jerárquica o Disciplinaria de las Fuerzas de Seguridad de la Provincia de Córdoba, se sancionó en 2014, en el marco de una serie de intentos de disciplinarizar a los miembros de la institución, luego del autoacuartelamiento de 2013.

14. Aquí se incorporó al personal de la casi desintegrada policía comunitaria, que era una dirección al interior de la Policía de Córdoba y que, para 2016, contaba con poco personal y un peso irrelevanteal interior de la institución.

15. La cantidad de módulos variaba según se tratara de personal subalterno o de líderes y supervisores. Los módulos comunes a ambas capacitaciones eran los siguientes: Introducción a la Policía Barrial, Sociología, Psicología Social, Investigación Preventiva, Adicciones, Derechos Humanos, Violencia Familiar, Derechos del Niño y del Adolescente, Uso Racional de las Armas, Uso Racional de la Fuerza, Actividad Física y Técnicas de Negociación.

16. Aspirante a agente es el nombre institucional para quienes están en formación en la Escuela de Suboficiales, mientras que cadete es el término utilizado para nombrar a quienes se están formando en la Escuela de Oficiales. 
el conocimiento sobre el trabajo de esta dependencia y aminorar las disputas entre las diferentes dependencias.

Esta particular inscripción al interior de la estructura organizacional de la Policía de Córdoba trae algunas implicancias para pensar los procesos de identificación de sus miembros. Por un lado, los corrimientos posibles con respecto a los efectivos de lo que los propios policías barriales suelen definir como la policía tradicional están signados por tener una estructura organizacional, una formación inicial y, en los casos de los(as) efectivos(as) con antigüedad, una trayectoria en la institución compartida con los miembros del resto de las dependencias. En este sentido, los miembros de la policía barrial tienen fuertes similitudes y pocos distanciamientos con respecto al resto de los efectivos de la institución. Como contracara de este piso común entre los miembros de la policía barrial y del resto de la institución, las transformaciones y disputas con el ideal policial que la postulación de un modelo de policiamiento comunitario o de proximidad puede tener no se ven reducidas exclusivamente a los(as) miembros de esta dependencia, sino que estos generan resonancias en toda la institución policial. Esto se debe, principalmente, a que la policía barrial trabaja de manera articulada con las demás dependencias y según las cuestiones de formación y trayectorias comunes antes mencionadas.

Las unidades territoriales de trabajo de la policía barrial son los cuadrantes, espacios geográficos en los que ha sido dividida la ciudad de Córdoba. Con base en variables socioeconómicas de los habitantes de esos espacios, los cuadrantes son clasificados en rojos, amarillos y verdes, siendo rojos los espacios de mayor vulnerabilidad, y verde, los de menos. En cada cuadrante se estipula el desarrollo de un consejo barrial, en el cual, mediante la figura del promotor barrial, se deben articular las demandas del sector con el gabinete interministerial ${ }^{17}$. De esto se desprende la intención de fomentar la interagencialidad, en pos de construir un nuevo tipo de vínculo entre el trinomio policía-comunidad-Estado. Aquí, siguiendo la clasificación de Sozzo (2008), se puede distinguir la voluntad de promover estrategias de prevención comunitarias, en la medida que se incentiva la participación ciudadana mediante los consejos barriales. Por su parte, existirían elementos relacionados con la prevención social, en tanto las falencias educativas, de servicios públicos y de infraestructura son entendidas como factores de riesgo a mitigar.

Entre las tareas de esta división se encuentra elaborar diagnósticos de los factores de riesgo operantes en el cuadrante y elevarlos al consejo barrial. Para este fin, 
se establece que los miembros de la policía barrial deben realizar entrevistas a los habitantes del barrio (Protocolo 5, anexos de Resolución 674). Asimismo, se estipula el diseño de rutas de patrullaje inteligente, diagramado a partir de la información obtenida del cuadrante. Para esto, la policía barrial diseñó su propia base de datos, en la que recopilaba información delictual proveniente de cuatro fuentes (llamadas al $101^{18}$, grupos de WhatsApp, entrevistas con vecinos(as) y denuncias en unidades judiciales). Esto se inserta en un proceso de crecimiento del trabajo realizado con información delictual de la mano de la apelación a discursos de eficiencia y reorganización policial. Por otro lado, se busca la gestión y mediación de conflictividades, partiendo del supuesto de que la intervención preventiva en los conflictos de convivencia puede evitar su desenvolvimiento violento o delictivo (Protocolo 10, anexos de Resolución 674). Se promueve entonces el paso de una gestión punitiva a una serie de estrategias de prevención urbana, social y ambiental (Anexo I, Resolución 674). Esto es mencionado, tanto por policías como por algunos actores pertenecientes a la Secretaría de Seguridad, como un policiamiento de proximidad, en contraste con el policiamiento reactivo o represivo.

\section{Cambios laborales y dignificación}

El Programa de Policía Barrial tiene como uno de sus objetivos la dignificación del trabajo policial, lo que implica la implementación de estrategias de bienestar policial. Respecto a esto, con base en el análisis de las entrevistas se considera que uno de los principales cambios en las condiciones laborales se vincula a la utilización y disponibilidad del tiempo policial. Aquí se destaca el establecimiento de turnos de trabajo de 8 horas y la delimitación de los recargos ${ }^{19}$ y adicionales. En principio, el personal de la policía barrial no deberá ser recargado y los adicionales deberán ser realizados en espacios que no impliquen una fuerte conflictividad ${ }^{20}$ (partidos de fútbol, boliches bailables, etc.). En este sentido, se facilita la delimitación entre el tiempo de trabajo y el tiempo privado. En esta línea, en varios relatos se comenta que la policía barrial tiene en cuenta las necesidades personales de cada efectivo a la hora de organizar el trabajo. Sobre esto, un cabo primero comenta:

18. Número de teléfono de emergencia en la Provincia de Córdoba.

19. Los recargos son horas extra no elegidas por el efectivo y no siempre pagadas como tales. Por su parte, los adicionales son turnos de horas extra aceptados de manera voluntaria por parte de los(as) policías.

20. Esto es en pos de que la labor policial realizada en los adicionales no afecte el perfil del efectivo como miembro de la policía barrial ni el vínculo construido con los(as) vecinos(as) del barrio. Se presupone que existen espacios de esparcimiento que requieren un policiamiento más represivo o de choque. 
Acá se les da, se los oye mucho, y yo les sé decir: "Agradezcan la líder que tienen que los escucha mucho" (...). Todos con un problema particular: se me murió el perro, se me murió el loro, mi hijo, mi hermano, mi abuelo, mi tío. Yo cuando entré a la policía eso no existía. "Tengo mi hijo enfermo", "Bueno que lo cuide la madre", te decían. Ahora le dan permiso, le dan todas las herramientas para que puedan cubrir bien el servicio. (Gustavo, comunicación personal, 25.10.2017)

La posibilidad de que las demandas individuales sean contempladas para la organización del servicio implica una modificación notable, ya que trastoca la jerarquía previamente establecida, por la cual primero estaría el servicio y la policía, y después cualquier otra cosa que pudiese importarle a esa persona. Esto viene a tensionar la idea de dedicación exclusiva que prima en algunas formas de ser policía. La misma entrevistada -también cabo primera- que en otra oportunidad había comentado no haber podido asistir al bautismo de su hijo por una demanda de un jefe, ahora destaca lo siguiente:

Sí, el líder ve a su gente como persona. Por ahí tiene contemplaciones, que en otro lugar (como en la guardia de infantería, ponele) no se tendría. Por ejemplo, en un duelo, se te muere tu papá. La policía te contempla tres días, pero acá a lo mejor te dan otro tiempo, en otro momento eso no le importaba a nadie. Porque el jefe ${ }^{21}$ piensa respecto al número, que tiene 10 efectivos, en cambio el líder considera qué puede dar la persona, porque sabe que vas a funcionar después mejor. No sirve de nada tener personal en la calle que está mal. La policía barrial es más humana. (Carla, comunicación personal, 14.11.2017)

Estas posibilidades van de la mano de la flexibilización de las relaciones jerárquicas y de la idea de escucha por parte de los(as) líderes. Estos cambios son descritos por un entrevistado de la siguiente manera:

Porque para cambiarle esa figura militar que tiene, porque el jefe, el jefe de compañía es muy militarizado, se cambió el nombre, entonces el jefe pasa a ser líder, líder barrial. El jefe de compañía pasó a ser supervisor, como si fuera una empresa, e incluso se sacó los borcegos de por dentro del pantalón y como ves nosotros lo usamos por fuera del pantalón, para que no tenga la imagen militar. Se trató de relajar la imagen de la policía (...). La verticalidad continúa existiendo. Pero, es como que acá bajó un cambio. De decir mal las cosas, en otro lado se les dicen mal las cosas: “andá, párate allá y dejá de joder”. Es así, en cualquier otra dependencia. “Eh, Juancito, ¿te podés instalar por favor en el operativo blanco acá a la vuelta?”. Se cambió la metodología de decir las cosas. (Gustavo, comunicación personal, 25.10.2017) 
Aquí se destaca el establecimiento de vínculos menos rígidos, junto con una serie de cambios institucionales que, según el entrevistado, buscan despegar a la policía barrial de la militarización del resto de la institución. A su vez, se ubica un cambio general de la imagen de los efectivos y de la policía barrial en su conjunto. En esta misma línea, otra entrevistada destaca también el impacto positivo del líder en el trabajo de uno de sus subordinados:

Roberto, por ejemplo, vos que lo conocés, no pegaba una: se esguinzaba, le pasaba esto, lo otro, un día tenía cáncer, al otro no. Y después se mejoró. Ahora él es primer ejemplo, anda en la moto chocho, hecho un... Y eso es por el líder, que te trata de otra forma, que trató de darle la vuelta cuando vio que no lo podía pasar de cuadrante. (Carla, comunicación personal, 14.11.2017)

En esta cita se distingue que, desde la interpretación de la entrevistada, las nuevas modalidades en los vínculos entre superiores y subalternos traen consigo una mejor gestión de los recursos humanos. Esto se condice con los objetivos en la Resolución 674 para el Programa de Policía Barrial, que establece como su objetivo general "optimizar el servicio de policía, fortaleciendo los recursos humanos, la delimitación territorial, la asignación de responsabilidades y la distribución eficiente de los recursos" (Anexo I).

No obstante lo dicho, algunos entrevistados destacaron la importancia de poner un limite a la flexibilidad, porque, pese a considerar positivamente los cambios, expresaron la necesidad de no perder la disciplina. Esto es expuesto por un entrevistado:

Y tuvimos una bárbara discusión hasta que le pudimos hacer entender que es así, y que podemos estar en la barrial que puede ser todo flexible pero que hay cosas que no se van a perder, que es el respeto a las canas, de la gente más vieja, a las jerarquías y a los estamentos policiales y a la función de cada uno. (Gustavo, comunicación personal, 25.10.2017)

Por otro lado, los(as) entrevistados(as) sostuvieron que en la policía barrial se gestaron nuevas formas de vinculación con los(as) vecinos(as). A este respecto, se señala, primeramente, la cercanía y confianza del vínculo. Sobre esto, en la encuesta aplicada a líderes y supervisores de la policía barrial en 2017, el $98 \%$ de los(as) encuestados(as) sostuvieron creer que la comunidad tenía una buena o muy buena imagen de la policía barrial (OPP, 2017b) 22. Esta cercanía es la que habilita diálogos

22. Este dato corresponde a una Encuesta realizada por el Equipo del Observatorio de Políticas Públicas de la Universidad Nacional de Villa María que trabajó por intermedio de un convenio con la Secretaría de Seguridad de la Provincia de Córdoba. La encuesta fue aplicada en noviembre de 2017 a todos(as) los(as) líderes y supervisores de la policía barrial. 
en los cuales los miembros de la policía barrial obtienen información relevante sobre delitos que se desarrollan en el barrio. En este aspecto, el sostenimiento de vínculos cercanos y fluidos con la comunidad es entendido como importante en tanto facilita el acceso a esa información conocida por los habitantes del sector, a la que solo se puede llegar mediante la confianza. Sobre esto, una cabo primera sostiene lo siguiente:

A mí me encanta la barrial. Es totalmente distinta a la policía que se conocía antes, de choque, que no daba explicaciones sobre el proceder. El vecino no era escuchado, ahora la verdad que yo creo que los vecinos están contentos y nos hablan. (Carla, comunicación personal, 14.11.2017)

Puede notarse que otro elemento que favorece este nuevo vínculo entre la policía y la comunidad es la explicación de motivos. Frente a una policía que actúa porque $s i$, la policía barrial se contrapone como una dependencia que puede exponer los motivos que guían su accionar. Sobre esto, un cabo primero se refiere a las tareas desarrolladas por otras dependencias que también patrullan, en contraste de lo que realiza la policía barrial:

Lo que hacen a diario es patrullar y parar a la gente con un cono. Parar en lugar sin motivo alguno, que van y le preguntan a la policía qué está haciendo acá o para qué está y no sabe para qué. Y qué es lo que debería hacer, debería dar una fundamentación desde la prevención, como hacemos nosotros que tenemos una fundamentación para la prevención. (Gustavo, comunicación personal, 25.10.2017)

Por último, se destaca que en varios relatos sobre la policía barrial se resalta su humanidad o la de sus jefes. Esto se torna singularmente relevante al tener en cuenta que muchas de las demandas por condiciones laborales que se vieron expresadas en el marco del autoacuartelamiento policial de 2013 podían condensarse en torno al significante humanidad. Sobre esto, un sargento dice:

El tipo [líder] se interesa en todo eso, es humano el tipo. Y eso es lo que le falta también a la policía y a muchos jefes, human... ser humano con las personas. Porque no somos robots. Somos padres, madres también a la vez... a la vez, es... un papá es madre y padre a la vez, o una mamá es madre y padre. A veces también tenemos nuestros problemas, tenemos nuestros... nuestras cosas internas también que... Entonces por ahí ser un poco humano y tratar de entender a esas personas es poner... es ser humano realmente. Creo que no somos... somos todos personas como vos, como tu viejo, como todas las personas, somos seres humanos. (Fernando, comunicación personal, 18.12.2017) 
De este modo, es posible observar cómo la incorporación a la policía barrial implicó para muchos de los(as) entrevistados(as) disputar algunos elementos del ideal policial. En primer lugar, se establece la preocupación por las condiciones laborales de quienes pertenecen a la dependencia, priorizando así el entendimiento de la labor en términos de trabajo, en contraste con la noción de vocación. En segundo lugar, se da una flexibilización de las relaciones jerárquicas que permite que las necesidades particulares sean tenidas en cuenta para la organización del servicio. Esto implica que no haya una primacía absoluta del tiempo policial por sobre el privado y que, en este marco, la línea divisoria entre ambos tiempos pueda ser sostenida por los(as) efectivos(as), aunque no sin tensiones. En tercer lugar, emerge en las entrevistas analizadas la idea de que en la policía barrial los(as) policías comprenden los motivos de la acción que están llevando a cabo y, en consecuencia, los podrían explicar si eso fuese demandado. Esto se contrapone al imaginario del efectivo obediente y disciplinado que realiza una tarea sin conocer la razón de la misma, ya que únicamente cumple órdenes. Por último, se resalta la idea de humanidad atravesada por la mayoría de los puntos anteriores. A este respecto, los(as) entrevistados(as) comentan que en la policía barrial se sienten considerados como personas, lo cual aparece de manera recurrente en el discurso policial como una forma de igualar a quien es policía con quien no lo es, en tanto uno y otro son personas.

En resumen, cabe mencionar que las nuevas lógicas laborales emergentes junto con la legitimación de un policiamiento distinto al tradicional implican una puesta en disputa del ideal policial.

\section{Alteridades y disputas entre policiamientos}

No obstante, estos cambios no han sido implementados sin disputas y resistencias. Sobre esto se destaca, en primer lugar, la fuerte rivalidad entre la policía barrial y el Comando de Acción Preventiva (CAP) ${ }^{23}$. Así, los miembros de otras dependencias con un perfil más reactivo emergen como la principal alteridad de los miembros de la policía barrial al ser cuestionados en torno al programa.

En la sistematización que se realizó sobre la implementación del Programa de Policía Barrial, se encontró que la dependencia con la que los miembros de la barrial tienen más conflictos es, sin lugar a dudas, el CAP (OPP, 2017b) ${ }^{24}$. Esto se debe a que

23. El CAP es un comando policial con perfil militarizado, creado en 2003.

24. Según los resultados de la encuesta previamente mencionada, la dependencia con la que más conflicto tienen es el CAP, seguida por el 101, el distrito y la comisaría. 
son las dos principales dependencias que patrullan en la actualidad, pero que, no obstante, tienen notables diferencia en lo que al tipo de policiamiento respecta. Sobre esto, es importante mencionar que, desde su creación en 2003, el CAP se había instalado como la dependencia con mayor presencia territorial mediante el patrullaje. Este cuasi monopolio es cuestionado recién con la implementación de la policía barrial.

Sobre esto, en uno de los informes internos del grupo de trabajo de la OPP-UNVM (2017a: 22), titulado "Informe 2-Capacitación de Líderes y Equipos de Gestión de la Policía Barrial", se ha expuesto que esta conflictividad puede explicarse teniendo en cuenta tres niveles:

En primer lugar, un nivel vinculado a "lo operativo", donde se ubican las discusiones por las comisiones, protocolos, límites territoriales y los recursos disponibles. En segundo lugar, conflictos "de convivencia", relacionados con el clima laboral y las relaciones interpersonales. Por último, identificamos un nivel de conflictividad referida a distintos policiamientos en disputa.

Por su parte, uno de los entrevistados se expresa de la siguiente manera:

Lo que sí, que la policía barrial tiene otro tipo de trabajo, o sea, es menos brusco, trata que a la noche si hay un hecho, un hecho de robo o un hecho de instancia privada, o un homicidio, un choque, un accidente, el personal de la barrial es un policía que está preparado para quedarse más con la persona, la víctima, el victimario o el que esté en ese momento, o el problema que tenga (...), entonces todo eso se acompaña mucho más que el policía común. No es que el policía común no lo haga, yo estuve trabajando en el CAP, pero qué hacemos en el CAP, el policía común antes: llegaba al hecho, identificaba el lugar donde estaba el robo, leía cómo era, hacía la inspección ocular del lugar, hacía un croquis del lugar y se iba, y entregaba el procedimiento en la parte judicial. (Fernando, comunicación personal, 18.12.2017)

En ese fragmento de la entrevista puede verse cómo el sargento distingue dos modos de abordajes diferentes a partir de la distinción entre los modos de intervención de ambas dependencias. En ese sentido, puede existir una disputa entre policiamientos que trae consigo una disputa sobre el ideal policial. Lo que está en juego es cuáles son las tareas que debe desempeñar un policía y cuál es su deber. Esto es particularmente relevante al tratarse de una labor en la cual las tareas a desarrollar suelen ser significadas en términos de misión-deber.

En adición, esta disputa entre policiamientos puede observarse también en la respuesta-pregunta a una de las cuestiones formuladas. Cuando se interrogó acerca de la principal tarea de la policía, la respuesta-pregunta de quienes pertenecían a 
la policía barrial era si esto se refería a la barrial o a toda la policía. La necesidad de separar a esta dependencia del resto da luces sobre la diferencia que inaugura la existencia de estos dos policiamientos. No solo suponen modos de intervención diferentes, sino que también conllevan objetivos diferentes. A esto se suma, como ha sido desarrollado, distintos modos de ser policía en la medida que difieren las aptitudes valoradas ${ }^{25}$.

Sin embargo, casi la totalidad de los(as)entrevistados(as) -tanto quienes pertenecen a la policía barrial como quienes no- entienden a estos dos policiamientos (reactivo y de proximidad) como complementarios o, al menos, como no excluyentes. Dentro de este registro, hay quienes comprenden que un policiamiento de proximidad es posible únicamente en barrios tranquilos. Habría entonces una división territorial y, en consecuencia, una separación de la población en dos grupos. El primero estaría conformado por barrios y habitantes tranquilos, es decir, de sectores medios y altos, en donde es posible y útil sostener un policiamiento de proximidad, ya que la conflictividad social general es baja, y los(as) vecinos(as) están dispuestos(as) a hablar con la policía. El segundo grupo estaría conformado por los sectores más peligrosos donde correspondería intervenir con un policiamiento reactivo-represivo. Así, estas dos formas son pensadas como complementarias, en términos locales-generales, pero se vuelven excluyentes en lo específico de cada territorio ya que existen sectores que no son compatibles con un policiamiento de proximidad.

Por otro lado, la lectura mayoritaria entre los(as) entrevistados supone a estos dos policiamientos como plenamente complementarios. Ya no a nivel general, correspondiéndole a algunos sectores un policiamiento de proximidad y a otros uno represivo, sino que en todos los sectores deberían coexistir. Esto es expresado por una entrevistada de la siguiente manera:

\begin{abstract}
Sí, igual está planeado que la policía barrial vaya a zonas de villas. Acá en Marques Anexo ${ }^{26}$, ya entró a algunas partes de la villa. Pero para que entremos nosotros primero tiene que pasar la policía de choque, y nosotros ir por atrás. (Carla, comunicación personal, 14.11.2017)
\end{abstract}

Sin embargo, más allá de la disputa sobre el ideal relacionado con el policiamiento de proximidad y los nuevos modos de ser policía a él asociados, existen notables persistencias de este ideal policial tradicional incluso entre quienes adscriben a la

25. A modo esquemático, se podría decir que aquí se valora la capacidad de empatía y diálogo, mientras que no son aptitudes excluyentes, por ejemplo, la fuerza y la valentía.

26. Nombre de un barrio popular en la zona norte de Córdoba. 
propuesta de la policía barrial ${ }^{27}$. Así, en el discurso de muchos de ellos es posible distinguir el modo en que las nociones de ser policía o actuar como policía siguen vinculadas a las maneras de actuar típicas del policiamiento reactivo:

Porque ahora es todo por cuadrante. Es una modalidad linda, pero también hay que tener policías que repriman. No es que nosotros no vamos a reprimir, llegado un momento si vos tenés que actuar como policía, vamos a actuar, somos policías. No es que somos una escuela aparte, ¿eh?, somos una rama de la policía, pero que está metida más en la sociedad, haciendo lo que antes hacía la policía social. (Fernando, comunicación personal, 18.12.2017)

Se puede diferenciar, primeramente, la complementariedad de los policiamientos, en tanto ambos son considerados necesarios. En segundo lugar, es posible visualizar las tensiones existentes respecto a la definición del actuar policial. Por un lado, el entrevistado sostiene que "si tenés que actuar como policía lo hacés", lo que supone que el accionar regular de la policía barrial está sometido a la realización de tareas que no serían las propias de la policía. Sin embargo, a continuación, remarca que "son policías" y que "son una rama de la policía". De este modo, la cita permite ver un terreno en disputa en el cual, se supone, algunos sentidos están en pleno proceso de sedimentación sin encontrarse aún consolidados.

En resumen, se considera que la incorporación a la policía barrial implicó para la mayoría de los(as) entrevistados(as) una fuerte dislocación en tanto puso en discusión qué significa ser policía, cuáles son las aptitudes que un policía debe tener y cuáles son los modos legítimos de intervención policial. Sin embargo, esta dislocación no implica necesariamente que estos procesos hayan decantado en una ruptura total con el ideal policial imperante.

Para cerrar, se destacan dos relatos considerados como significativos para dar cuenta de estas disputas:

Entonces vos empezabas a tener un miedo por dentro. Porque afuera vos tenés miedo, no es que seamos robots, somos personas, porque tenemos que tener miedo. Porque el miedo lo que te hace es retraerte a cualquier cosa o cualquier... vos entrás a una casa y te... el miedo te hace... ¿eh?... te resguarda un poco la vida tuya. Y uno, eh... en

27. A este respecto, quienes se oponen al modelo propuesto por la policía barrial suelen sostener posturas que reivindican el policiamiento represivo, a la vez que resaltan varios de los elementos descritos del ideal policial tradicional, como los atributos legítimos para ser un buen policía. Entre estos se destacan la valentía, el arrojo, la disciplina y la obediencia. Asimismo, critican a la policía barrial por considerarla marketinera y que responde a objetivos políticos, donde el interés estaría más en mejorar la imagen de la institución, mostrando una policía en movimiento y activa, que por la problemática del delito en sí. 
ese momento uno actuaba bajo presión. Por eso me fui también a la policía barrial. (Fernando, comunicación personal, 18.12.2017)

Pero bueno, yo toda mi vida he luchado para ser yo, para ser diferente dentro de la policía, manteniendo mi esencia. Nunca quise dejar de ser femenina, eso de ir al choque así a lo macho a míno me gusta. Y me di cuenta que en la policía barrial era un poco eso, y pensé cómo no fue así cuando yo entraba. (Carla, comunicación personal, 14.11.2017)

En el primer fragmento se pone en evidencia el modo en que la policía barrial viene a significar un contraste con la dependencia anterior, a partir de ubicar al miedo como punto central de un ideal policial que premiaba la valentía y el arrojo por sobre el resguardo personal. Por su parte, en la segunda cita la entrevistada expone que en su trayecto en la policía tuvo que luchar para "conservar su esencia", contraria al ideal policial masculinizado. En este contexto, la policía barrial se muestra como un espacio en el que no son necesarios aquellos modos y aptitudes que ella no tenía ni deseaba tener.

Así, retomando la clave teórica propuesta a lo largo del trabajo, se sostiene que en este proceso es posible distinguir atisbos de un desplazamiento en la significación que articula al significante policía. Como se ha expuesto, aquí se observa una disputa en torno a los modos legítimos de intervención policial y las aptitudes necesarias para ser un buen policía. De esta manera, este contexto puede ser pensado como un momento de desestabilización de la identidad previamente objetivada.

\section{Conclusiones}

Con base en lo expuesto, se considera que la implementación de la policía barrial trae consigo fuertes transformaciones para quienes comenzaron a formar parte de esta dependencia, especialmente en lo referido a las condiciones laborales. Por un lado, la existencia de una organización del tiempo de trabajo policial que incorpora las necesidades de los(as) policías les permite sostener cierta escisión entre los tiempos policiales y los privados. Esto, a su vez, implica que la vida familiar/personal y la policial no deban ser concebidas, necesariamente, como excluyentes. En este sentido, se va en contra de la tendencia totalizante u omnipresente que aparece vinculada al Estado policial y su regulación de los tiempos. De este modo, se habilitan algunos corrimientos hacia una concepción de la labor policial más afín a la idea de trabajo.

Por otro lado, la flexibilización de las jerarquías y la humanidad de los jefes posibilitan el sostenimiento de vínculos laborales menos disciplinarizados. Asimismo, las nuevas formas de vinculación con los(as) vecinos(as) promueven una labor policial que no debe solo obedecer órdenes, sino ser capaz también de explicar los motivos 
de su accionar. Estos elementos, bajo la idea de la dignificación laboral, fomentan el entendimiento de la labor policial en términos de trabajo.

Así, las nuevas lógicas laborales de la mano de la incorporación del policiamiento de proximidad permiten poner en discusión qué significa ser policía, cuáles son las aptitudes que se deben tener y cuáles son los modos legítimos de intervención. En este aspecto, la policía barrial implica una disputa en torno al ideal policial.

Por su parte, respecto al policiamiento de proximidad, se destaca como principal alteridad a los miembros de otras dependencias más militarizadas, teniendo en cuenta que entre ellas existen disputas operativas, de convivencia y en torno a los modos de policiamiento.

En conclusión, este hito logra poner en tensión el ideal policial tradicional de distintas maneras. Sin embargo, esto inaugura un terreno pantanoso en el cual los sentidos en torno al ser policial se encuentran aún levemente sedimentados. En este sentido y tal como se ha desarrollado, coexisten elementos del ideal policial tradicional junto con otros rasgos que lo ponen en disputa, pudiendo incluso articularse de diversas formas.

\section{Referencias}

Aboy-Carlés, Gerardo (2001). Las dos fronteras de la democracia argentina. Buenos Aires: Homo Sapiens.

Carbajo, Mariana (2016). La educación policial y el gobierno de la seguridad en la provincia de Córdoba. Reformas en la formación policial, policialización de la seguridady modelo tradicional de policía (2003-2013) [Tesis de maestría]. Universidad Nacional de Córdoba, Córdoba.

Carbajo, Mariana; Rodríguez, Florencia; Goldin, Déborah; Buzzeti, Andrés (2017) Modelo policial y políticas de seguridad: aproximaciones a la organización de la Policía de Córdoba entre los años 2007 y 2015. Trabajo presentado en III Jornadas de Sociología de UnCuyo. Mendoza, Argentina. Recuperado de:http://bdigital.uncu.edu.ar/objetos_digitales/10402/ modelopolicialypolticasde-.pdf

Castro, Sofía; Castro, Julián; Goldin, Déborah (2016). De prácticas policiales y legalidades: una aproximación a pensar la policía de Córdoba y el nuevo Código de Convivencia Ciudadana. Trabajo presentado en II Congreso de la Asociación Argentina de Sociología, Villa María, Argentina. Recuperado de http://catalogo.unvm.edu.ar/doc_num.php.

Crisafulli, Lucas; Castro, Julián (2017). La metamorfosis de la seguridad en Córdoba: entre el actuarialismo y el delito de los pobres. Trabajo presentado en I Jornadas de estudios sociales sobre delito, violencia y policía. La seguridad en cuestión, Ciudad de La Plata y Quilmes, 
Argentina. Recuperadode http://www.memoria.fahce.unlp.edu.ar/trab_eventos/ev.10366/ ev.10366.pdf

Dammert, Lucía (2007). Seguridad pública en América Latina: ¿qué pueden hacer los gobiernos locales? Nueva Sociedad, 212, 67-81. Recuperado de https://nuso.org/media/articles/ downloads/348o_1.pdf

Equipo técnico de becarios del Observatorio de Políticas Públicas de la Universidad Nacional de Villa María en la Secretaría de Seguridad de la Provincia de Córdoba (2017a). Informe 2. Capacitación de líderes y equipos de gestión de la Policía Barrial. Inédito.

Equipo técnico de becarios del Observatorio de Políticas Públicas de la Universidad Nacional de Villa María en la Secretaría de Seguridad de la Provincia de Córdoba (2017b). Informe 7. Resultados de encuesta a la Policía Barrial. Inédito.

Frederic, Sabina (2009). En torno a la vocación policial y el uso de la fuerza pública: identidad y profesionalización en la policía de la provincia de Buenos Aires. En La policía en perspectiva histórica. Argentina y Brasil (del siglo XIX a la actualidad) (s.p.), editado por Ernesto Bohoslavsky; Lila Caimari; Cristiana Schettini. Buenos Aires: Universidad de San Andrés.

Foa-Torres, Jorge (2016). Posmarxismo y análisis de políticas públicas: fundamentos ontológicos, bases epistemológicas y estrategias metodológicas para el estudio de políticas desde la teoría política del discurso. POSTData, 2O(2), 355-385. Recuperado de http://www. revistapostdata.com.ar/2016/o1/posmarxismo-y-analisis-de-politicas-publicas-fundamentos-ontologicos-bases-epistemologicas-y-estrategias-metodologicas-para-el-estudio-de-politicas-desde-la-teoria-politica-del-discurso-post-marxis/

Galvani, Mariana (2016). Cómo se construye un policía: la Federal desde adentro. Buenos Aires: Siglo XXI.

Garriga-Zucal, José (2016). El verdadero policía y sus sinsabores. Esbozospara una interpretación de la violencia policial. La Plata: Universidad de La Plata.

Garriga-Zucal, José; Melotto, Mariano (2013). La diversidad (in)visible. Identidad(es) entre policías bonaerenses. Avá, 22, 77-96.

Goldin, Déborah (2019). Ser policía: lógicas identitarias y alteridades. Un análisis de los procesos de identificación de los/as suboficiales de la Policía de la Provincia de Córdoba (2013-2017) [Trabajo final de grado]. Universidad Nacional de Villa María, Córdoba.

Hathazy, Paul (2013). (Re)militarización policial en Argentina: entre luchas burocráticas y lógicas políticas. Trabajo presentado en XIV Congreso Nacional y Latinoamericano de Sociología Jurídica, Córdoba, Argentina.

Kessler, Gabriel (2011). El sentimiento de inseguridad. Sociología del temor al delito. Buenos Aires: Siglo XXI. 
Lorenc-Valcarce, Federico (2009). Orden, derechos y delitos. La inseguridad en la agenda pública argentina. El Príncipe, 3(2), 83-107

Monjardet, Dominique (2010). Lo que hace la policía. Sociología de la fuerza pública. Buenos Aires: Prometeo.

Reiner, Robert (1992). The Politics of the Police. Oxford: Oxford University Press.

Saín, Marcelo (2008). El Leviatán azul:policíay política en la Argentina. Buenos Aires: Siglo XXI.

Seri, Guillermina (2011). Discrecionalidad policial y ley no escrita: gobernando en el estado de excepción. En Mirada (de) uniforme. Historia y crítica de la razón policial (pp.349-380), coordinado por Diego Galeano; Gregorio Kaminsky. Buenos Aires: Teseo.

Sirimarco, Mariana (2009). De civil a policía: una etnografía del proceso de incorporación a la institución policial. Buenos Aires: Teseo.

Sozzo, Máximo (2008). Inseguridad, prevención y policía. Quito: FLACSO.

Sozzo, Máximo (2014). Delito común, inseguridad y respuestas estatales: inercia e innovación durante la década kirchnerista a nivel nacional en Argentina. Cuestiones de Sociología, 10. Recuperado de http://www.memoria.fahce.unlp.edu.ar/art_revistas/pr.6330/pr.6330.pdf

Sozzo, Máximo (2016). Postneoliberalismoy penalidad en América del Sur. Buenos Aires: CLACSO. 